\title{
Mobility Modeling for Personal Networks
}

\author{
Yanying Gu • R. Venkatesha Prasad • Ignas Niemegeers
}

Published online: 25 December 2009

(C) The Author(s) 2009. This article is published with open access at Springerlink.com

\begin{abstract}
A Personal Network (PN) is a user-centric design interconnecting numerous devices belonging to a user in different geographic locations, such as home, office, car, etc., to form a single global network to offer fully personalized services. In PNs devices of the user move in different groups, where these groups merge and split. In this paper, we design and simulate a PN Mobility Model (PNMM) to capture the characteristics of movements of devices in PNs. We propose a simple stability evaluation method for group mobility models and apply the method to PNMM. Through the stability evaluation, we find that PNMM possesses long-term steady state behavior. Moreover, for the evaluation of mobility models, some evaluation methods have been proposed, which include non-homogenous node mobility, relative node mobility in a group, and dynamics of group merging and splitting. Analysis shows that PNMM is better than other models to represent the PN mobility properties. In addition, the impact of PNMM on the performance of a PN Clustering Protocol (PNCP) has been studied. Simulation results provide insights into the performance of PNCP, and provide guidelines for future design of PN clustering. PNMM can be easily applied to any Personal Area Network or Body Area Network with slight modifications.
\end{abstract}

Keywords Mobility model · Personal Networks · PN mobility model · Stability analysis $\cdot$ PN clustering protocol

Y. Gu $\cdot$ R. V. Prasad $(\bowtie) \cdot$ I. Niemegeers

Center for Wireless and Personal Communications (CWPC), Delft University of Technology,

Delft, 2628 CD, The Netherlands

e-mail: vprasad@ewi.tudelft.nl; rvprasad@ieee.org

Y. Gu

e-mail: y.gu@ewi.tudelft.nl

I. Niemegeers

e-mail: i.niemegeers@ewi.tudelft.nl 


\section{Introduction}

Ubiquitous computing (ubicomp) [24] described by Mark Weiser-the father of ubiquitous computing —indicates that the computing power will be embedded into smart devices, rooms, clothing and even on our bodies. From a few devices per person, in the near future there will be hundreds or even thousands of devices per person per office. Freescale's CTO Lisa Su during the technical keynote at the Freescale Technology Forum (FTF) [4] also stated that there will be 1,000 embedded devices per person in 2015 . With the development of embedded intelligence and systems, these embedded devices collect data, process it and also connect to each other. Su predicts that by 2015 core processors will be fabricated at $22 \mathrm{~nm}$. Systems on chip would have gone wafer scale by then. Thus a large number of parallel computing systems would have been evolved down to the personal devices, which have wireless connectivity. When the networking function is ready, these 1,000 devices around a person will be invisible. People simply use them without knowing them. These devices will work autonomously, individually and collectively. We need to control and integrate thousands of devices for each person in a scalable way. This could be achieved by a Personal Network (PN) $[1,16,18]$ —a user-centric network - controlling the user's devices, data streams and interactions with other users on a global scale.

A Personal Network (PN) $[1,16,18]$ enables a private and secure network to interconnect various personal devices to support a person with all his communication needs-anywhere and anytime. To enable the environments involving many devices per person in the future, PN addresses some challenges: self-configuration, self-healing, self-optimization, etc., while interacting with the outside environment (or other networks or persons) [1,16,18]. A PN is schematically represented in Fig. 1 to provide an overview. Through a PN, the user can ubiquitously access personal or public services. Considering the design of the network beyond the existing computational infrastructure, many devices should be connected and controlled by a PN for a user. A PN extends the geographical scope of a Personal Area Network (PAN), within the range of a few meters around the person to a global one. Starting from the scope of a PAN, a PN covers different locations where devices of a person reside, such as, home, office, vehicle, class room, etc., as shown in Fig. 1. In a PN, devices that are separated by hundreds of kilometers can form a single network-with the help of infrastructure networks-in which devices cooperate with each other to support personal communications. Thus these devices may be involved in both, short-range communications using Bluetooth, IR, WLAN, etc., and middle/long-range communications using GPRS, UMTS networks, etc. Interconnecting these personal devices poses big challenges for PN networking design.

Personal Nodes are communicating devices that are owned by the user and are used for supporting the person with many services. They use various types of networks, such as, the Internet, WPAN, WLAN, UMTS, etc. They may not be always interconnected. Moreover, technical issues such as service/session mobility, self-organization, context awareness, service discovery and management, etc., $[1,16,18]$ of a PN are far more complex that a PAN is not able to support and provide these higher levels of performance. Since a PN will include thousands of devices per person (in future) in various networks and at different places, the mobility of these personal devices challenges PN design, such as, seamless services, session continuity, context aware services and its discovery, security, etc., [1,16,18].

The behavior of these devices needs to be addressed from the mobility point of view and it should be close to the reality. Mobility models are divided into two categories: generic and realistic. Generic mobility models $[2,19]$ capture node movement with stochastic methods, where all the nodes behave identical to each other statistically and should maintain a stable state over time [25]. Random waypoint [12], random walk [20], and Gauss-Markov 
[2] mobility models are all examples of generic mobility models. Realistic mobility models $[11,14]$ describe real scenarios and capture real movement characteristics. In this paper, we propose a new mobility model for PNs. The reasons for a new model are: (a) Actual mobility traces of thousands of devices per person are not available at present. The dense PN scenarios, involving thousands of devices per person per room, have not been studied. The difficulties of the measurement of these dense PNs come from the limitation of the current technology, which cannot support thousands of devices per person [4]. Without actual mobility traces of personal nodes, mobility issues in PNs can only be studied, possibly, using a generic model at this moment. (b) The performance results of the protocols proposed in the literature are highly dependent on the underlying mobility models [2]; it may work well with one model, but perform poorly with another. Thus, a mobility model representing the movement patterns of the devices or personal nodes of a PN is necessary to evaluate the protocols developed for PNs. (c) The scenarios under consideration in this paper are different from many other cases in the literature. Currently in the literature, it is assumed that a single user or vehicle carries one mobile device. Some mobility scenarios of ad hoc networks have been studied, where persons (each carrying a mobile device) move in an area [11,14]. However, in our work, many embedded devices move together with the user or vehicle. The scenario studied in this paper will be useful in future and it is necessary in view of node and group movement.

Basically, we need to study whether the proposed techniques and protocols perform well in the typical PN scenarios. A mobility model representing the behavior of nodes in PN scenarios is thus required. A PN includes devices not only around the user in the range of PAN, but also can contain more devices in various locations, such as home, office, vehicle, etc., as shown in Fig. 1. Covering various places, a PN includes many devices per person per room. Thus, to support the user's needs, a PN consists of a core PAN and various remote groups of devices, which can be in various locations; see Fig. 1. In PN scenarios, many embedded devices per person form a PAN and move in a group with the user. As the user leaves home, devices in the proximity of the user in home split into two groups: one group is still at home; the other group goes with the user similar to a PAN. As he arrives at the work place, two groups of devices (one group, which is the PAN, and the other group, which is in the office) merge together to form a single group in the office. This shows that the groups of

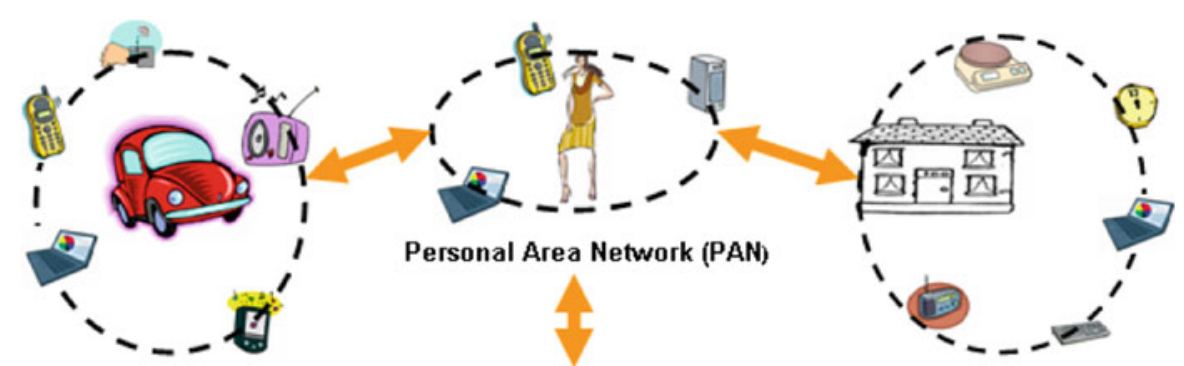

PAN \& Vehicle Area Network

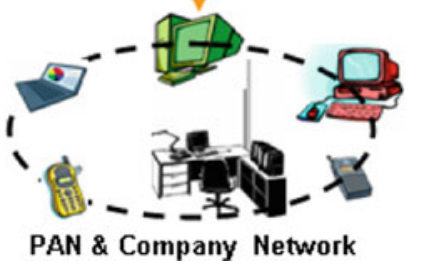

Home Network \& PAN

Fig. 1 Personal Network 
the devices have dynamics including moving, splitting and merging. The behavior of these groups of personal nodes influences the interconnection of a large number of PN devices and challenges the services offered by a PN. Thus it is important to study the mobility issues of PNs.

Rest of the paper is organized as follows. In Sect. 2 some typical PN scenarios are studied and the PN mobility properties have been discussed. Then some group mobility models from the literature are explained in Sect. 3. In Sect. 4 our PN mobility model (PNMM) is explained. The stability evaluation of PNMM is in Sect. 5. In Sect. 6 the analysis of the PNMM and other existing mobility models. In Sect. 7 the simulation results are given, where PNMM significantly influences the protocol evaluation for PNs. Finally in Sect. 8, we summarize our work and discuss our future plans.

\section{PN Applications and its Mobility Properties}

Before we study PN applications and its mobility properties, formally we provide the context of two important terms here: (a) personal node: a personal node is a personal device that can communicate with other nodes or devices. In this paper, for all practical purposes we make no distinction between the devices and personal nodes and use them interchangeably. Further, we use the terms personal node and node synonymously. (b) group: a group is a collection of a number of personal nodes that are in the close vicinity and are able to communicate with each other. A group of nodes can be wearable devices around a person's body, or personal devices in the same location, such as home, office, car, etc. A group could be immobile in some cases, for example, a PC and a printer in the house.

A mobility model for a PN should capture majority of features of movements of nodes in a PN. Since PN is a user-centric design to support personal applications, typical PN scenario user cases are to be examined first to get the key characteristics of mobility in a PN. The ideas of user-centric design can be found in the literature such as, "Telecom Scenarios in 2010" [7], "The Book of Visions: Visions of the Wireless World" [3], "Scenarios for Ambient Intelligence in 2010" [5]. Some detailed scenarios of a PN are proposed in My Personal Adaptive Global NET (MAGNET) [18]. Scenarios and services of a PN cover the following aspects: shopping mall, education, travel, community, work, emergency, health care, entertainment, transportation, etc. [18]. The distribution and behavior of the nodes highly depend on the services and applications offered by a PN. We introduce in this section some PN services and applications for various scenarios. We draw the mobility patterns of nodes in a PN through these PN services and applications. We list here the applications that can capture the most of the mobility characteristics. We get the requirements (nine requirements in total, R1-R9) for PN mobility model here.

\subsection{A Health-Monitoring Application}

Monitoring the health conditions of a disabled or an elderly person is a potential personal service of a PN [4]. It can collect useful data not only for emergency situations but also for daily health monitoring. Thus a PN incorporates sensing and actuating devices linked to a health-monitoring server at home. Some sensing devices are installed in various places at home to collect raw data for health applications. The user takes some wearable nodes with him/her moving along with the user. Thus we write the first Requirement as, R1: some personal nodes never move, and some can move. 
While a person moves from one room to another, some wearable and mobile nodes move in a group with the person for the purpose of monitoring the user's health. Therefore, we write $\mathrm{R} 2$ as: personal nodes cooperate to support the demands of a user, they are not independent, and usually move in groups.

\subsection{Business Environment}

The ideal personal work environment expected by a business person is that he can perform any task anywhere using available tools [4]. Considering the physical environment of a person, such as his home, office, and car, a person has some groups of personal nodes at different places. A businessman has a number of personal nodes at different places for social and private needs. While the businessman with his PAN moves between these places, the number of personal nodes at each place varies. Thus we write R3: the number of personal nodes in each group is not fixed. In addition, the coverage areas of these places are also different since the devices are heterogeneous and may use different radio technologies. When groups of nodes move in these places, the group coverage area is also different. Thus we write R4: the coverage area of each group is not fixed, and we can set a maximum value.

As the businessman leaves his office and enters his car, some personal nodes are left in the office; other personal nodes are carried by him and are moved into the coverage area of the on-board car network. Thus R5 is: group of nodes can split. When the person moves into his car-while he is in a conference call using his PDA- then the PN can enable the conference call seamlessly by handing over to the on-board car network, where on-board speakers, microphones and the PDA are used together to offer the conference call service. Therefore we write R6: two groups of personal nodes can merge into one group.

\subsection{A Remote Babysitting Application}

In case of a mother keeping a vigil on her sleeping baby, her PN supports her needs by dividing her personal nodes into various groups according to her demands [3]. For this application, the mother can divide her nodes into two groups: one group is in the room, monitoring her baby; and the other group of nodes carried by her to display pictures of her baby. Thus R7 is: the number of groups of personal nodes can be pre-set. When the mother moves, the speed of the group of personal nodes with her is within a maximum value. Therefore we list R8 as: the group speed is same for the member nodes, and can be limited by a maximum value. After an hour, when her baby is awake, the two groups merge into one. While the mother does house work in the same room as her baby, she needs to watch her baby at the same time, and uses the baby monitoring service. In this room, all nodes form a group. When the mother moves, the nodes with her also move w.r.t. the nodes on and near her baby; this results in R9: the personal nodes included in the same group can have relative mobility with each other.

Based on these examples of the use cases and scenarios, we found nine requirements for PN mobility model and are listed in Table 1. These nine requirements are used in the design and evaluation of PN mobility model in the later sections. We do not proclaim that the set of these nine requirements are exhaustive and suffice all the scenarios. However, we believe this set covers the most frequent and common use cases. This set only forms an important basis for the analysis in the following sections. However, we acknowledge ameliorating these requirements when new use-cases are found. 
Table 1 PN mobility requirements

Requirements

1 The mobility heterogeneity of personal nodes: some personal nodes never move, some can move

2 Personal nodes cooperate to support the demands of a user, they are not independent, and usually move in groups

3 The number of personal nodes in each group is not fixed

4 The coverage area of each group is not fixed, and we can set a maximum value

5 Group of nodes can split

6 Two groups of personal nodes can merge into one group

7 The number of groups of personal nodes can be pre-set

8 The group speed is same for the member nodes, and can be limited by a maximum value

9 The personal nodes included in the same group can have relative mobility with each other

\section{Related Work}

The movement of persons has been modeled by mobility models [14], which represent their indoor and outdoor social lives. Some experiments [11] have been done to collect the real data of movements of humans. Unlike the research on the mobility of a large group of persons, our work focuses on the movement of various nodes that supports the needs of a user. Since the nodes in a PN cooperate to satisfy the user, the mobility characteristics of these nodes are different from that of the movement of a person. We describe many group mobility models $[10,17]$ in this section. We examine the extent to which the existing solutions can be used to model mobility in PNs with the requirements listed in the previous section. The mobility models proposed until now are used as a coarse benchmark and we compare our model with them to gain an insight.

\subsection{Reference Point Group Mobility Model (RPGM)}

RPGM models the group mobility, where the movement of a group is represented by the logical center of the group moving along a series of pre-defined check points [15]. In each group, an individual mobile node moves around its reference point with a value derived from a random motion vector. However, this model assumes that a group of nodes always move together, thus it does not meet the PN mobility model requirements R5 and R6.

\subsection{Reference Region Group Mobility Model (RRGM)}

RRGM is designed for search or rescue scenarios that a group of rescuers move towards an affected spot/destination $[16,17]$. It defines a reference region as a destination to which a group of mobile nodes move. Since an individual rescuer may rest, go slow, etc., some nodes arrive at the destination region early, which should wait for the late-arriving nodes. Clearly PN scenarios explained in the previous section are different from the scenarios considered in RRGM. A group of personal nodes in PNs should move together to satisfy the needs of a user. Thus RRGM is not suitable for PN mobility modeling. 
Fig. 2 Movement of a group

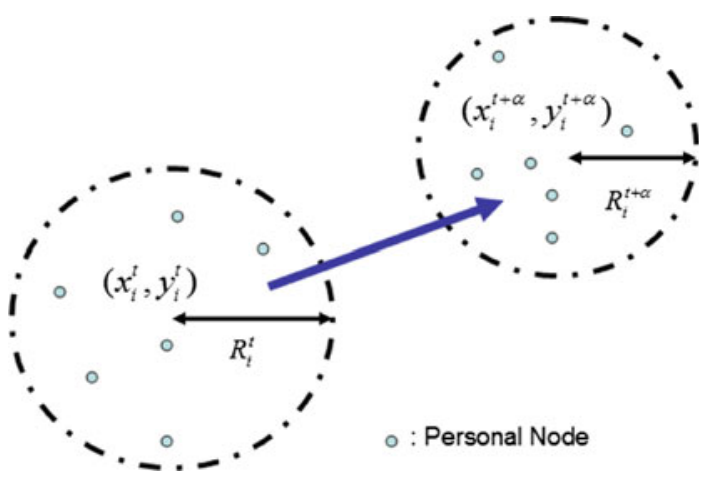

Different mobility models listed above typically focus on a specific scenario. Although some mobility models can meet a part of PN mobility requirements, none of them meet all the listed requirements. Thus a new mobility model for a PN is required and has been addressed here.

\section{PN Mobility Model}

An earlier version of PNMM has been presented in [9]. We extend the description of PNMM with more details. We explain it in the following five steps. The principles of PNMM to meet each of PN mobility requirements (R1-R9) are also highlighted.

\subsection{Step 1: Initialization}

In order to meet the PN mobility Requirement R1, there are $n_{s}$ static nodes, $n_{m}$ mobile nodes, and in total $N$ nodes. The positions of $n_{s}$ static personal nodes are chosen randomly in the simulation area. At $t=0, n_{m}$ mobile nodes are divided into $M$ groups (Eq. (2)), which present the mean value of the maximum and minimum number of groups represented by $X$ and $Y$.

$$
\begin{aligned}
& N=n_{s}+n_{m} \\
& M=(X+Y) / 2
\end{aligned}
$$

Each node selects the group to join in the vicinity and this meets the Requirement R3. As shown in Fig. 2, in the $i$ th group, $n_{n, i}^{(t=0)}$ personal nodes stay in a circular region with the center, $\left(x_{i}^{(t=0)}, y_{i}^{(t=0)}\right)$, which is chosen randomly in the simulation area of length $L$ and width $W$. The group covers a circular area with a radius $r_{i}^{(t=0)}$, which is chosen randomly in the range $\left(0, r_{\max }\right]$. The coverage area of the group is chosen such that it satisfies the Requirement R4. In addition, the location of a node $\mathrm{j}$ in group $\mathrm{i}\left(x_{i, j}^{(t=0)}, y_{i, j}^{(t=0)}\right)$ is chosen randomly in the region as shown on the left side of the Fig. 2.

\subsection{Step 2: Movement of a Group}

The movement of a group is changed at the beginning of each time interval $\alpha$, and represented by the movement of the central reference point of the group. The central reference 
point randomly selects a direction $\theta$ from the range $[0,2 \pi)$ and the speed from $\left[0, V_{\max }\right]$. By choosing the speed of the central reference point of a group, $v_{g}^{(t)}$, for $g^{\text {th }}$ group, from $\left[0, V_{\max }\right]$, the speed of a group can be limited within a maximum value, which meets the Requirement R8. Thus the central reference point moves from the position $\left(x_{i}^{(t)}, y_{i}^{(t)}\right)$ to $\left(x_{i}^{(t+\alpha)}, y_{i}^{(t+\alpha)}\right)$ in time $\alpha . d_{i}^{(t, \alpha)}$ is the distance travelled by the $i$ th group in interval $\alpha$, and it can be calculated as,

$$
d_{i}^{(t, \alpha)}=\sqrt{\left(x_{i}^{(t)}-x_{i}^{(t+\alpha)}\right)^{2}+\left(y_{i}^{(t)}-y_{i}^{(t+\alpha)}\right)^{2}} .
$$

The radius, $r_{A}^{(t+\alpha)}$ of the group coverage area, at the end of the time interval $\alpha$, is chosen randomly from $\left(0, r_{\max }\right]$. With the center $\left(x_{i}^{(t+\alpha)}, y_{i}^{(t+\alpha)}\right)$ and radius $r_{A}^{(t+\alpha)}$, the destination area of a group is chosen as shown in Fig. 2. For each node in the group such as a node $j$ in group $i,\left(x_{i . j}^{(t)}, y_{i . j}^{(t)}\right)$, it randomly selects a point $\left(x_{i . j}^{(t+\alpha)}, y_{i . j}^{(t+\alpha)}\right)$, which is in the group destination area, and moves towards it with a constant speed of $v_{i, j}^{(t)}$, which is given by,

$$
v_{i, j}^{(t)}=\sqrt{\left(x_{i, j}^{(t)}-x_{i, j}^{(t+\alpha)}\right)^{2}+\left(y_{i, j}^{(t)}-y_{i, j}^{(t+\alpha)}\right)^{2}} / \alpha
$$

Thus all the personal nodes in a group move together at the same time, which meets the Requirement R3. On the other hand, the nodes in the same group have relative random mobility, because their positions are chosen randomly inside the group coverage area at $t+\alpha$ to meet the PN mobility model Requirement R9.

\subsection{Step 3: Group Merging}

To meet the PN mobility model Requirement R6 in Table 1, group merging is planned at each time interval $\alpha \times c$, where $c=1,2,3, \ldots$. When $c=1$, the group merging interval is the same as the group movement interval $\alpha . c$ controls the frequency of group merging activity. Since $n_{g}^{(t)}$ groups of nodes randomly move with a maximum speed $V_{\max }$, and if two groups are near each other, they can merge into one group in the time interval $\alpha$. Thus the group merging requirement is formulated as given in Eq. (5), where the distance, $d_{a, b}$ between the two central reference points of groups $a$ and $b$ will be,

$$
d_{a, b}=\sqrt{\left(x_{a}^{(t)}-x_{b}^{(t)}\right)^{2}+\left(y_{a}^{(t)}-y_{b}^{(t)}\right)^{2}} \leq 2 V_{\max } \alpha
$$

If groups $a$ and $b$ meet the group merging requirement as specified in Eq. (5), where the centers of two groups can meet in the next time interval within the maximum speed, $V_{\max }$, then group $a$ and group $b$ can merge. The center of the new group $A,\left(x_{A}^{(t+\alpha)}, y_{A}^{(t+\alpha)}\right)$, is randomly chosen in the overlapping area of the two circles as shown in the Fig. 3, where the two circles meet. The radius of group $A, r_{A}^{(t+\alpha)}$ in the next time interval, $t+\alpha$, is chosen randomly in the range of $\left(0, r_{\max }\right]$. Here we simply follow the same set of rules as given in Step 1 and Step 2, where the radius is chosen to be in $\left(0, r_{\max }\right]$. The argument is that when two groups meet, it is not necessary that combined area increase. If a person walks into his car, the area remains almost the same. In fact in some cases it may shrink since there could be merging and division at the same time (Step 5). With the chosen center reference point $\left(x_{A}^{(t+\alpha)}, y_{A}^{(t+\alpha)}\right)$ and group radius $r_{A}^{(t+\alpha)}$ of the target group $A$ as demonstrated in Fig. 3, 


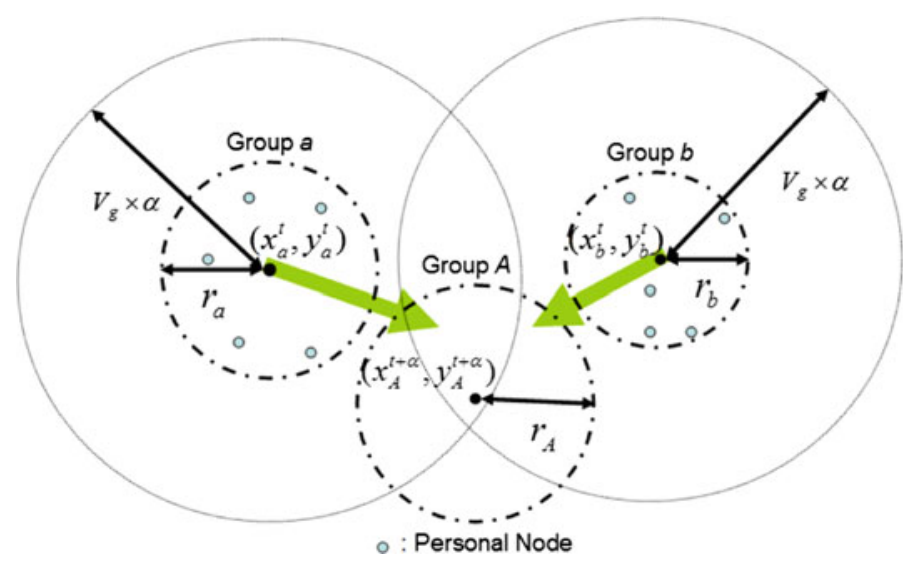

Fig. 3 Group merging

the group $a$ and $b$ moves into the coverage area of the new group $A$, which is described as follows: for a node $j$, in group $a$, it starts from point, $p_{s}=\left(x_{a, j}^{(t)}, y_{a, j}^{(t)}\right)$, randomly selects a point $p_{d}$ in the new merged group $A,\left(x_{A, j}^{(t+\alpha)}, y_{A, j}^{(t+\alpha)}\right)$; in the time interval $\alpha$, starting from $p_{s}$, the node $j$ moves with a constant speed to $p_{d}$.

Based on the group merging requirement, we can get $n_{m p}^{(t)}$ pairs of groups that have the possibility of merging. $n_{g}^{(t)}$ is the current number of groups. To ensure the number of groups to be always greater than the minimum number of groups $Y$, the maximum number of pairs of groups to be merged, $m$, is,

$$
m=\operatorname{Min}\left(n_{m p}^{(t)},\left(n_{g}^{(t)}-Y\right)\right)
$$

The number of pairs of groups to be merged, $n_{m}^{(t)}$, is chosen based on normal distribution in the range of $(0, m) \cdot n_{m}^{(t)}$ pairs of groups are randomly chosen amongst $n_{m p}^{(t)}$ pairs of groups having the possibility to be merged. Thus the number of pairs of groups to be merged, $n_{m}^{(t)}$ is

$$
n_{m}^{(t)} \leq\left(n_{g}^{(t)}-Y\right)
$$

After group merging, $n_{m}^{(t)}$ groups disappear. Thus, based on Eq. (7), in the next time interval $t+\alpha$, the number of groups, $n_{g}^{(t+\alpha)}$, will be higher than $Y$, which is the lower limit on number of groups (i.e., between $X$ and $Y$ ).

\subsection{Step 4: Group Division}

To meet the Requirement R5, the group division is considered at every time interval $\alpha \times d$, where $d=1,2,3, \ldots$, as shown in Fig. 4 . When $d=1$, the group merging interval is same as the group movement interval $\alpha . d$ controls the frequency of group division. Group $a$ can be divided into two groups, where group $a$ is the original group and group $k$ is the new group after group division in the next time interval $t+\alpha$. The destination areas of groups $a$ and $k$ are chosen similarly by following the rules described in Step 2. Each node in the original group $a$ can randomly choose the group it joins; group $a$ or $k$. Then each node moves towards the destination group area. 


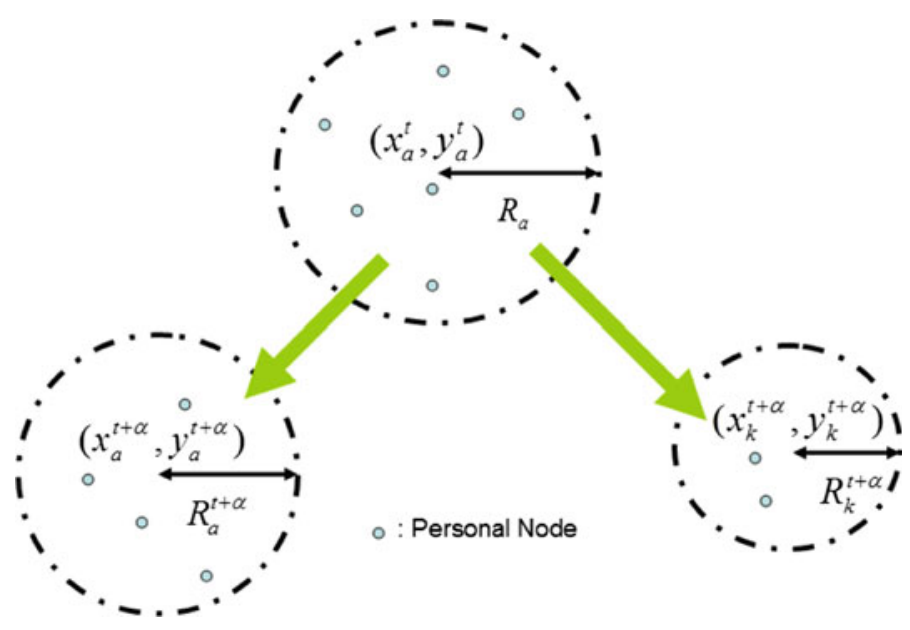

Fig. 4 Group division

Every group has the possibility to be divided into two groups. For the number of group to be divided, $n_{d}^{(t)}$, should be randomly chosen in the range of $\left(0,\left(X-n_{g}^{(t)}\right)\right)$.

Then $n_{d}^{(t)}$ groups are randomly chosen from $n_{g}^{(t)}$ groups. Thus,

$$
n_{d}^{(t)} \leq\left(X-n_{g}^{(t)}\right)
$$

The number of groups in the next time interval, $n_{g}^{(t+\alpha)}$, will be lower than the upper limit of number of groups, $X$.

\subsection{Step 5: Group Merging and Division at the Same Time}

For group $a$ chosen to be both merged with group $b$ and divided, first division is considered. Those nodes chosen to be included in group $k$ will move to group $k$ (following Step 4). Other nodes will be merged with group $b$ (following Step 3).

We note that the upper and lower limits $X$ and $Y$ are used here to constrain the possible fluctuations in the number of groups. Without any limit, the topology of the network may vary drastically which is counter productive for the formulation of group mobility model in PNs. However, by choosing $Y$ to be one and $X$ to be the number of nodes itself, one can generalize this model. Thus the limits used here generalize the model attempted.

\section{Stability Evaluation}

Group mobility is a typical kind of movement of nodes in many scenarios, such as portable nodes moving with the user to support his needs [9], fire-fighters rescuing in an emergency situations [2]. In group mobility scenarios, mobile nodes belonging to a group have similar movement characteristics, while the nodes related to different groups have diverse mobility patterns. These frequently occurring group movements have been modeled earlier [2].

Before using mobility models in the performance evaluation, the mobility models have to be verified, because the performance of mobility models influences the evaluation of 
protocols at every step in the simulation. Stability evaluation for a novel mobility model, random waypoint (RWP) mobility model has been studied and some surprising results have been reported in [25]. For RWP, nodes move randomly and behave identically. So it is believed that the node-average performance, such as average node speed should be constant w.r.t the simulation time. However, evaluation results of RWP [15,25] show that its average node speed decreases gradually to zero, when time tends to $\infty$. RWP cannot guarantee the same average node speed over a long period resulting in incorrect simulation results $[15,25]$.

For group mobility models, the question is whether they offer stable performance (high stability) with time. We define the stability of group mobility models as follows: a group mobility model is stable, if its performance parameters, such as number of groups, average group speed, and average node speed, etc., maintain the same values as simulation time tends to infinity. In this section, we study the stability issues for group mobility models and our contributions are three-fold: 1) since no extensive study of stability for group mobility models has been done, we firstly discuss the necessities, and challenges of stability evaluation for group mobility models; 2) the stability evaluation methods including analysis and simulation are proposed for all generic ad hoc group mobility models; 3 ) the proposed stability evaluation methods are applied to PNMM [17] as an example of stability evaluation. Although, we give stability evaluation results of only PNMM, the methodology of stability evaluation can be used for all generic ad hoc group mobility models.

\subsection{Stability Evaluation of Group Mobility Models: Necessities and Challenges}

Whether the performance of a mobility model fits the planned design target is a key point to be tested. According to the group behavior mobility models for ad hoc networks can be categorized into two kinds: individual node mobility models, and group mobility models. Individual node mobility models give solutions for the movement of each mobile node with less correlation with regard to other nodes in the domain. Random waypoint (RWP) mobility model [2] is an example of a generic mobility model. Group mobility models cover both group movement from a global perspective, and movement of nodes inside each group from a local perspective. To study the performance of mobility models, we start from a relatively simple case of individual node mobility models. Individual node mobility covers three aspects: 1) position, 2) speed, and 3) direction. In [2], generic individual node mobility models have been proposed providing the rules for calculating the position, speed and direction. Usually, researchers are interested in node-average and time-average performance in the evaluation of mobility models. For generic individual node mobility models, node-average speed at each instant of time is $\overline{V(t)}=\frac{1}{N}\left(\sum_{i=1}^{N} V_{i}(t)\right)$, where $N$ is the number of nodes and $V_{i}(t)$ is the instant speed of node $i$; and time-average speed $\bar{V}=\int_{t=0}^{T} \overline{V(t)} d t$, where $T$ is the simulation time. Usually, $T$ is considered to be very large to test the long-run behavior of mobility models. Thus a generic individual node mobility model is steady, only if the instant node-average speed, $\overline{V(t)}$, maintains the same value as that of $\bar{V}$ when time tends to $\infty[15,25]$.

For group mobility, group movement, individual node movement and relative node movement inside each group are modeled. The evaluation covers both node and group levels. At the node level, node-average performance metrics are tested, which are the same as in the evaluation of individual node mobility models. On the group level, the group-average performance metrics are of interest. For stability evaluation, generic group mobility models throw some challenges: 
(a) The dynamics of instant group speed: Generic group mobility models present the behavior of groups. For a group which includes a number of nodes, usually, nodes in the same group have relative mobility, where the distance between nodes change as the group moves. Usually, in group mobility models $[9,10]$, the instant speed of a group $V_{g}(t)$ is represented by the center of the group. The instant speed of the center of a group is taken as the instant group speed. Another way is taking the node-average speed in a group as the group speed. It is not sure yet which is better.

(b) Group level mobility: In generic ad hoc group mobility models, groups move randomly. Globally, on the group level, the group-average speed is specified as $\overline{V_{g}(t)}=$ $\frac{1}{n_{g}^{(t)}}\left(\sum_{i=1}^{n_{g}^{(t)}} V_{g}^{i}(t)\right)$, where $n_{g}^{(t)}$ is the number of groups and $V_{g}^{i}(t)$ is the instant speed of group $i$. Group-average speed, $\overline{V_{g}(t)}$, at time $t$ represents the instant group mobility. The group-average speed should be maintained, which may be the median of the maximum and minimum group speeds. We define the requirement for the steady state of group mobility model as the instant group-average speed varying around a constant value, say $\bar{V}$. Whether a group mobility model is steady at every phase during simulation is to be answered by the stability evaluation.

(c) Relative mobility inside a group: Nodes move in groups and have relative mobility inside a group. The relative node mobility in a group is usually modeled as the relative speed between a node and the center of a group [2,9]. Thus nodes in a group have dynamic relative distance, speed and direction of movement when they move with the group. How to evaluate the mobility inside each group is a critical question for generic group mobility model evaluation.

(d) Dynamics of group merges and splits: For a group mobility model, its dynamics are characterized by not only the movement of nodes, but also the group merging and splitting. A group mobility model performs poorly, if the number of groups tends to one (in case of more merges than splits) or the number of nodes (more splits than merges). The number of groups will be changing during the simulation. Here we add another requirement for the steady state for group mobility model: if the $n_{g}^{(t)}$ is the number of groups at time $t$, the average number of groups, $E\left[n_{g}^{(t)}\right]$, should remain constant as simulation time tends to infinity.

In our stability evaluation we investigate the generic group mobility model that can reach a steady state. Based on the challenges and requirements for group mobility model, and stability evaluation presented in the above paragraphs, following performance metrics are considered in the stability evaluation for ad hoc group mobility models: the average speed of groups and the average speed of nodes to test the stability from the point of view of groups and nodes, and the average number of groups to address the stability of the group merging and splitting.

Can PNMM maintain a steady state? Is PNMM steady at $t=0$ ? When can PNMM reach the steady state? How long does it last? Do the performance values vanish? These questions will be answered by stability evaluation as explained in the further sections.

\subsection{Stability Analysis}

Some terminologies used in this section are listed first. Let $R$ be the distance traveled by the center of a group in each time interval $\alpha ; v_{g}$ is the average group speed; $v_{n}$ is the average node speed; $\overline{n_{g}}$ is the average number of groups. Other terms used in this section are the 


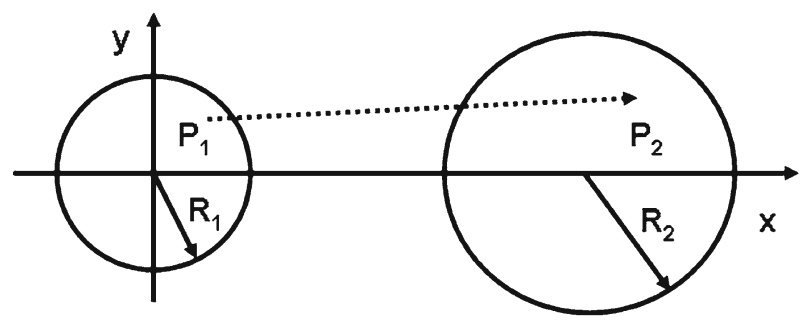

Fig. 5 Node movement inside group

same as in Sect. 4. For the analysis of average speed of groups and nodes, we first consider the situation without group merging and splitting. For the scenarios with group merging and splitting, the performance is studied by simulations.

\subsubsection{Average Group Speed}

In PNMM, the instant group speed is represented by the speed of the center of a group [9]. Since the speed of a group is chosen in each time interval $\alpha$ uniformly in $\left[0, V_{\max }\right]$, the probability density function of group speed is $f_{v}(v)=1 / V_{\max }$. Thus the probability density function of $R$, which is the distance travelled by the center of a group in each time interval $\alpha$ is $f_{R}(r)=1 /\left(\alpha V_{\max }\right)$. Thus $E\left[v_{g}\right]=\int_{0}^{V_{\max }} v f_{v}(v) d v=V_{\max } / 2$. The average group speed is $V_{\max } / 2$.

\subsubsection{Average Node Speed $v_{n}$}

Under the rules of PNMM in each time interval $\alpha$, a group moves from the original group coverage area to the destination group coverage area, which is shown in Fig. 5. For an individual node, it moves from $P_{1},\left(x_{1}, y_{1}\right)$, randomly selected in the original group coverage area, to $P_{2},\left(x_{2}, y_{2}\right)$, randomly selected in the destination group coverage area as shown in Fig. 5 .

Considering all the nodes in each group, we calculate the average distance traveled by the nodes in each time interval as the average length between $P_{1}$ and $P_{2}$ as follows:

$$
\begin{aligned}
\left|\overrightarrow{P_{1} P_{2}}\right| & =E\left(\sqrt{\left(x_{1}-x_{2}\right)^{2}+\left(y_{1}-y_{2}\right)^{2}}\right) \\
& =\sqrt{E\left(x_{1}^{2}+x_{2}^{2}+y_{1}^{2}+y_{2}^{2}-2 x_{1} x_{2}-2 y_{1} y_{2}\right)} \\
& =\sqrt{\left(E\left(x_{1}\right)\right)^{2}+\left(E\left(x_{2}\right)\right)^{2}+\left(E\left(y_{1}\right)\right)^{2}+\left(E\left(y_{2}\right)\right)^{2}-2 E\left(x_{1} x_{2}\right)-2 E\left(y_{1} y_{2}\right)}
\end{aligned}
$$

In Fig. 5, $x_{1}$ and $x_{2}$ are independent, so as $y_{1}$ and $y_{2}$, thus $E\left(x_{1} x_{2}\right)=E\left(x_{1}\right) E\left(x_{2}\right)$; $E\left(y_{1} y_{2}\right)=E\left(y_{1}\right) E\left(y_{2}\right) ; E\left(x_{1}\right)=0 ; E\left(x_{2}\right)=V_{\max } \alpha / 2 ; E\left(y_{1}\right)=0 ; E\left(y_{2}\right)=0$. Thus $\left|\overrightarrow{P_{1} P_{2}}\right|=E\left(x_{2}\right)=V_{\max } \alpha / 2$ and the average node speed, $v_{n}$, is $V_{\max } / 2$, as the simulation progresses.

\subsubsection{Average Number of Groups}

Since the group merging and splitting are modeled by PNMM, the number of groups change with time. The group merging requirement is the distance between two groups $i$ and $j$ being $d_{i, j}=\alpha V_{\max }$. The probability of any two groups being within a distance $R_{\max },\left(R_{\max }=\right.$ 
$\left.\alpha V_{\max }\right)$ in a square of side $L$, is $P=\pi R_{\max }^{2} / L^{2}$. Since groups are uniformly placed in the area under consideration, there are $P\left(n_{g}^{(t)}-1\right)$ neighbor groups, where $n_{g}^{(t)}$ is the current number of groups, within the distance $R_{\max }$. The number of pairs of groups that can be merged are $P\left(n_{g}^{(t)}-1\right) n_{g}^{(t)} / 2=\pi R_{\max }^{2}\left(n_{g}^{(t)}-1\right) n_{g}^{(t)} /\left(2 L^{2}\right)$.

According to the group merging rule of PNMMEq. (7), if $\pi R_{\max }^{2}\left(n_{g}^{(t)}-1\right) n_{g}^{(t)} /\left(2 L^{2}\right) \geq$ $n_{g}^{(t)}-Y$ (we call this Scenario-1 hereafter), the number of merged pairs is uniformly chosen in $\left[0,\left(n_{g}^{(t)}-Y\right)\right]$; otherwise, if $\pi R_{\max }^{2}\left(n_{g}^{(t)}-1\right) n_{g}^{(t)} /\left(2 L^{2}\right)<n_{g}^{(t)}-Y$ (called Scenario-2 hereafter), the number of merged pairs is uniformly chosen in $\left[0,\left(\pi R_{\max }^{2}\left(n_{g}^{(t)}-1\right) n_{g}^{(t)} /\left(2 L^{2}\right)\right)\right]$. Thus the number of groups is always higher than $Y$.

\subsubsection{Scenario-1}

This scenario is explained as follows:

$$
\begin{aligned}
& \frac{\pi R_{\max }^{2}\left(n_{g}^{(t)}-1\right) n_{g}^{(t)}}{2 L^{2}} \geq n_{g}^{(t)}-Y, \quad \pi R_{\max }^{2}=A ; L^{2}=B \\
& {\left[\frac{1}{2}\left(1+\frac{2 B}{A}\right)\right]^{2}-\frac{2 B}{A} Y \leq\left[n_{g}^{(t)}-\frac{1}{2}\left(1+\frac{2 B}{A}\right)\right]^{2}}
\end{aligned}
$$

If $\left[\frac{1}{2}\left(1+\frac{2 B}{A}\right)\right]^{2}-\frac{2 B}{A} Y \leq 0$, for all $n_{g}^{(t)}$ (the current number of groups) the above eqnarray holds. Thus,

$$
Y \geq \frac{B}{2 A}+\frac{A}{8 B}+\frac{1}{2}
$$

If $\left[\frac{1}{2}\left(1+\frac{2 B}{A}\right)\right]^{2}-\frac{2 B}{A} Y>0$, based on Eq. (10), $n_{g}^{(t)} \geq \sqrt{\left[\frac{1}{2}\left(1+\frac{2 B}{A}\right)\right]^{2}-\frac{2 B}{A} Y}+\frac{1}{2}\left(1+\frac{2 B}{A}\right)$ or $n_{g}^{(t)} \leq \sqrt{\left[\frac{1}{2}\left(1+\frac{2 B}{A}\right)\right]^{2}-\frac{2 B}{A} Y}-\frac{1}{2}\left(1+\frac{2 B}{A}\right)$. Since, $\sqrt{\left[\frac{1}{2}\left(1+\frac{2 B}{A}\right)\right]^{2}-\frac{2 B}{A} Y}-$ $\frac{1}{2}\left(1+\frac{2 B}{A}\right)<0$ we only consider $n_{g}^{(t)} \geq \sqrt{\left[\frac{1}{2}\left(1+\frac{2 B}{A}\right)\right]^{2}-\frac{2 B}{A} Y}+\frac{1}{2}\left(1+\frac{2 B}{A}\right)$.

Because $Y \leq n_{g}^{(t)} \leq X$; thus,

$$
X \geq \sqrt{\left[\frac{1}{2}\left(1+\frac{2 B}{A}\right)\right]^{2}-\frac{2 B}{A} Y}+\frac{1}{2}\left(1+\frac{2 B}{A}\right) .
$$

In this scenario, in the $i$ th time interval, the number of merged pairs, $d_{i}$, is uniformly chosen in $\left[0,\left(n_{g}^{(t)}-Y\right)\right]$; the number of groups to be split, $c_{i}$, is uniformly chosen in $\left[0,\left(X-n_{g}^{(t)}\right)\right]$. The probability density function of $d_{i}$ is specified below,

$$
f_{d}=1 /\left(n_{g}^{(t)}-Y\right)
$$

thus its expectation is $E[d]=\left(n_{g}^{(t)}-Y\right) / 2$.

Similarly, the probability density function of $c_{i}$ is specified below,

$$
f_{c}=1 /\left(X-n_{g}^{(t)}\right)
$$

thus its expectation is $E[c]=\left(X-n_{g}^{(t)}\right) / 2$. 
Let $\overline{n_{i}}$ be the number of groups in the $i$ th time interval. In the first interval it is set as $\overline{n_{1}}=(X+Y) / 2$. In the $(i+1)$ th time interval $\overline{n_{i+1}}=\overline{n_{i}}+c_{i}-d_{i}$. The average number of groups,

$$
\overline{n_{g}}=\sum_{i=1}^{k} \overline{n_{i}} / k=\left[(X+Y) / 2+\sum_{i=1}^{k-1}\left(\overline{n_{i}}+c_{i}-d_{i}\right)\right] / k
$$

From Eqs. (13) and (14), $\sum_{i=1}^{k-1} c_{i}=(k-1) \frac{X}{2}-\frac{1}{2} \sum_{i=1}^{k-1} \overline{n_{i}}$ and $\sum_{i=1}^{k-1} d_{i}=\frac{1}{2} \sum_{i=1}^{k-1} \overline{n_{i}}-$ $(k-1) \frac{Y}{2}$. Thus

$$
\overline{n_{g}}=\frac{1}{k}\left[\frac{X+Y}{2}+\sum_{i=1}^{k-1} \overline{n_{i}}+\sum_{i=1}^{k-1} c_{i}-\sum_{i=1}^{k-1} d_{i}\right]=\frac{X+Y}{2}
$$

For Scenario- 1 : if either $Y \geq \frac{B}{2 A}+\frac{A}{8 B}+\frac{1}{2}$ or $X \geq \sqrt{\left[\frac{1}{2}\left(1+\frac{2 B}{A}\right)\right]^{2}-\frac{2 B}{A} Y}+\frac{1}{2}\left(1+\frac{2 B}{A}\right)$, the average number of groups remains at $\overline{n_{g}}=(X+Y) / 2$ as expected. Thus the stability in terms of number of groups in Scenario- 1 is satisfactory.

\subsubsection{Scenario-2}

Different from Scenario-1 explained in Eq. (10), Scenario-2 is specified as follows:

$$
\begin{gathered}
n_{g}^{(t)}-Y>\frac{\pi R_{\max }^{2}\left(n_{g}^{(t)}-1\right) n_{g}^{(t)}}{2 L^{2}} \\
{\left[\frac{1}{2}\left(1+\frac{2 B}{A}\right)\right]^{2}-\frac{2 B}{A} Y>\left[n_{g}^{(t)}-\frac{1}{2}\left(1+\frac{2 B}{A}\right)\right]^{2}>0}
\end{gathered}
$$

Thus $\left[\frac{1}{2}\left(1+\frac{2 B}{A}\right)\right]^{2}-\frac{2 B}{A} Y>0$ and

$$
Y<\frac{B}{2 A}+\frac{A}{8 B}+\frac{1}{2}
$$

Because of Eq. (17), $0 \leq n_{g}^{(t)}<\sqrt{\left[\frac{1}{2}\left(1+\frac{2 B}{A}\right)\right]^{2}-\frac{2 B}{A} Y}+\frac{1}{2}\left(1+\frac{2 B}{A}\right)$ and $Y \leq n_{g}^{(t)} \leq X$, thus $Y<\sqrt{\left[\frac{1}{2}\left(1+\frac{2 B}{A}\right)\right]^{2}-\frac{2 B}{A} Y}+\frac{1}{2}\left(1+\frac{2 B}{A}\right)$. For Scenario-2 in the $i$ th time interval, the number of merged pairs, $c_{i}$, is uniformly chosen in $\left[0, \pi R_{\max }^{2}\left(n_{g}^{(t)}-1\right)^{2} / 2 L^{2}\right]$; the number of groups to be split, $d_{i}$, is uniformly chosen in $\left[0,\left(X-n_{g}^{(t)}\right)\right]$. Thus $E[c]=$ $\left[\pi R_{\max }^{2}\left(n_{g}^{(t)}-1\right)^{2}\right] \mathrm{Big} / 4 L^{2}, E[d]=\left(X-n_{g}^{(t)}\right) / 2$.

The average number of groups, $\overline{n_{g}}$, is

$$
\begin{aligned}
\overline{n_{g}}= & \frac{Y+X}{2}+E[d]-E[c] \\
= & \frac{1}{k} \sum_{i=1}^{k} \overline{n_{i}}=\frac{Y}{2}+\frac{1}{k}\left[\frac{1}{2} \sum_{i=1}^{k} \overline{n_{i}}+\sum_{i=1}^{k}\left[Z\left({\overline{n_{i}}}^{2}-2 \overline{n_{i}}+1\right)\right]\right] \\
& \text { where, } Z=\frac{\pi R_{\max }^{2}}{4 L^{2}}
\end{aligned}
$$



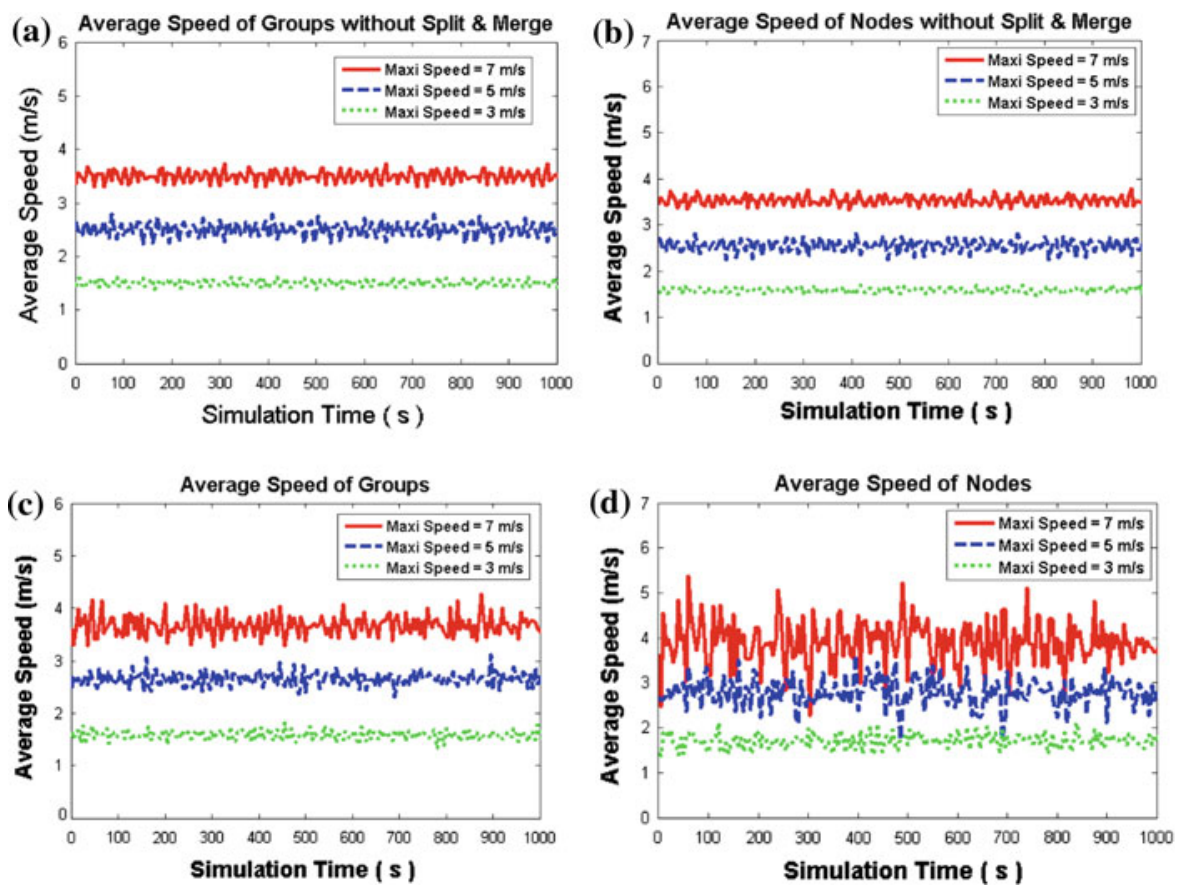

Fig. 6 Stability simulation results of average speed of group and average speed of nodes

If $\overline{n_{i}}=X$, when $k \rightarrow \infty,{\overline{n_{g}}}_{\max }=X-Z(X-1)^{2}$. If $\overline{n_{i}}=Y$, when $k \rightarrow \infty,{\overline{n_{g}}}_{\min }=$ $(X+Y) / 2-Z(Y-1)^{2}$. Since, $\bar{n}_{g_{\min }} \leq \overline{n_{g}}$ and $Y \leq X$,

$$
Z=\frac{\pi R_{\max }^{2}}{4 L^{2}} \leq \frac{X-Y}{2\left[(X-1)^{2}-(Y-1)^{2}\right]}
$$

Thus, for Scenario-2 if Eq. (20) holds, average number of groups is not $(X+Y) / 2$. For example, if we set $X=10, Y=1, \sqrt{9 \pi / 2} R_{\max } \leq L$. So a large $L$ results in average number of groups which is not $(X+Y) / 2$. The simulation results in the next subsection will show how the average number of groups changes in case of a large $L$, as the simulation area increases.

\subsection{Study of Stability Using Simulation}

PNMM is implemented as a mobility generator producing mobility files for simulations. The parameters for PNMM are set as follows: the maximum number of groups is 10 ; the minimum number of groups is 5; the number of nodes is 200 ; the simulation area is $100 \mathrm{~m} \times 100 \mathrm{~m}$; the group radius is $10 \mathrm{~m}$. We run PNMM 10 times and its mean conformed to $95 \%$ confidence level.

Since the center of a group is chosen randomly in the simulation area in each time interval $\alpha$, which can be reached within a maximum value $v_{\max }$. PNMM is expected to maintain constant average speed of groups and average speed of nodes as the simulation progresses. Figure 6 shows the average speed of groups and the average speed of nodes. Table 2 gives the average speed and their deviation in our experiments corresponding to the results in Fig. 6. 
Table 2 Stability simulation results of average group speed and average node speed

\begin{tabular}{|c|c|c|c|c|c|c|}
\hline \multirow{2}{*}{$\begin{array}{l}\text { Parameters } \\
V_{\text {max }}\end{array}$} & \multicolumn{3}{|c|}{ Without split and merge } & \multicolumn{3}{|c|}{ With split and merge } \\
\hline & $3 \mathrm{~m} / \mathrm{s}$ & $5 \mathrm{~m} / \mathrm{s}$ & $7 \mathrm{~m} / \mathrm{s}$ & $3 \mathrm{~m} / \mathrm{s}$ & $5 \mathrm{~m} / \mathrm{s}$ & $7 \mathrm{~m} / \mathrm{s}$ \\
\hline$v_{g}$ & 1.4982 & 2.5043 & 3.4984 & 1.5854 & 2.6534 & 3.6673 \\
\hline STD & 0.0630 & 0.1313 & 0.1073 & 0.0882 & 0.1317 & 0.1960 \\
\hline$v_{n}$ & 1.5860 & 2.5513 & 3.5311 & 1.7115 & 2.7855 & 3.8975 \\
\hline STD & 0.0552 & 0.1334 & 0.1022 & 0.1554 & 0.3177 & 0.5038 \\
\hline
\end{tabular}

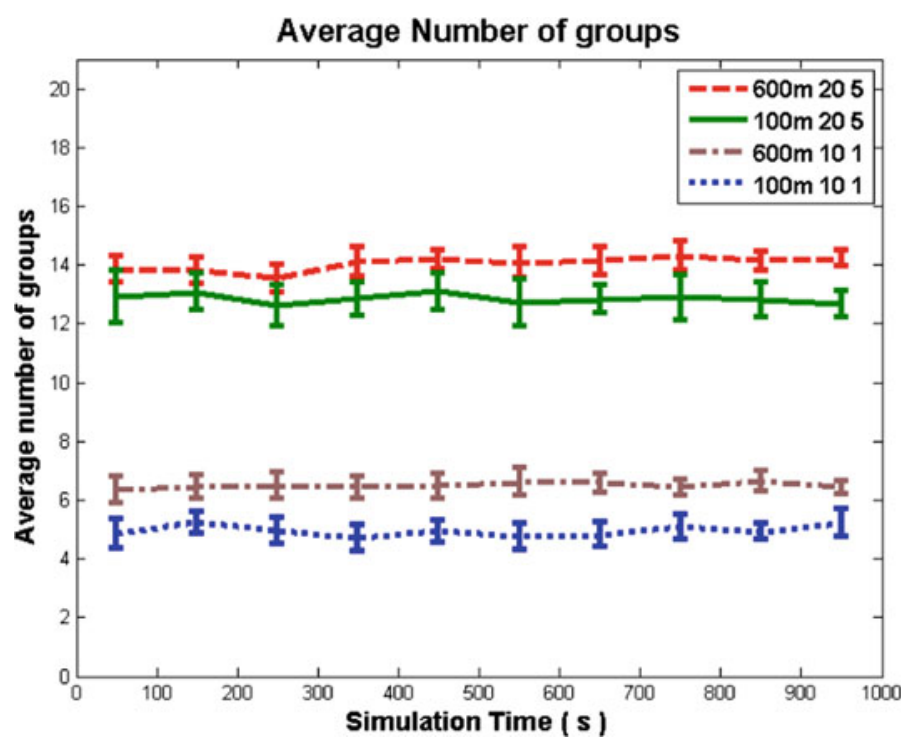

Fig. 7 Stability simulation results of average number of groups

Starting from the simple initial conditions, without group merging and splitting, the average speed of groups and the average speed of nodes are shown in Fig. 6a and b and Table 2. The results support the analysis in the previous section. The average speed of groups and the average speed of nodes vary around a consistent value, which is $V_{\max } / 2$.

Considering group merging and splitting, the average speed of groups and the average speed of nodes are shown in Fig. 6c and d and Table 2. Because group merging and splitting are added in the simulation, both the average speed of groups and nodes are higher than $V_{\max } / 2$. Since nodes in the same group move with the center of the group, the speed of the group influences the speed of nodes. Thus, the speed of nodes changes and is higher than $V_{\max } / 2$. The deviation is also higher. The average speed of nodes is more fluctuating than that of the groups because of the design principle of PNMM (see Sect. 4, Step 1 and Step 2) that the nodes in the same group have relative mobility too.

The long-run behavior of the group merging and splitting can be addressed by calculating the average number of groups during simulation. In Fig. 7, the parameters used for PNMM are: the number of nodes is 200 ; the group radius is $10 \mathrm{~m}$; and the maximum speed is $3 \mathrm{~m} / \mathrm{s}$. Average number of groups is calculated every $100 \mathrm{~s}$. We ran the simulation 15 times. 
In Fig. 7, the parameters for each set of simulation reads as follows: for example, for the simulation experiment represented by the first line from top, the area is $600 \mathrm{~m} \times 600 \mathrm{~m}$; maximum number of groups is 20 , minimum is 5; similarly, for the third line from top, the area is same; but maximum number of groups is 10 , minimum is 1 . As shown in Fig. 7, the first line and third line corresponds to Scenario- 1 and the second and forth line corresponds to Scenario-2 explained in Sect. 5.2. An increase in area from $100 \mathrm{~m} \times 100 \mathrm{~m}$ to $600 \mathrm{~m} \times 600 \mathrm{~m}$ results in the increase in average number of groups. The stability analysis, in the previous section, shows that a larger $L$ (Eq. 20) results in the average number of groups being not equal to $(X+Y) / 2$. The results in Fig. 7 confirm and match with the analysis. Moreover, these results provide further inferences as to how the average number of groups change as the simulation area increases. We may infer that a larger operational area (larger $L$ ) results in higher average number of groups. With the increase in time, the average number of groups for each $\alpha$ stays around a constant in all scenarios.

From the analysis and simulation results in the above two sub-sections, we get some properties of stability of PNMM. We summarize them here.

- Without group merging and splitting, the average speed of groups, $v_{g}$, and the average speed of nodes, $v_{n}$, can maintain a consistent value, which is half of the maximum speed, $V_{\max } / 2$.

- With group merging and splitting, $v_{g}$ and $v_{n}$ also maintain a consistent value, which is higher than $V_{\max } / 2$.

- The average number of groups $\overline{n_{g}}$ consistently varies around a value in all the scenarios.

- Simulations are in a limited area, specified by $L$, and $\overline{n_{g}}=(X+Y) / 2$.

- Simulations in a larger area beyond a threshold, i.e., as $L$ increases, $\overline{n_{g}}$ is greater than $(X+Y) / 2$.

Thus, from the above results, we see that PNMM has a steady long-run behavior and provides a steady state in the beginning and throughout the simulation as well. This behavior makes it an ideal choice for group mobility model for simulating closely knit networks such as PANs.

\section{Performance Comparison of Group Mobility Models}

For the evaluation of mobility models, some evaluation methods are proposed to test the group mobility models in this section. We evaluate RPGM [10], RRGM [17], and PNMM [9]. We test which one is best suited for PNs in terms of the PN mobility model Requirements (R1-R9) in Table 1. The evaluation methods are divided into four categories: (1) the heterogeneous node mobility (R1); (2) group mobility and node mobility inside a group (R2, R4, R8, R9); (3) group merging \& splitting (R5, R6); (4) number of groups and number of nodes in each group $(\mathrm{R} 3, \mathrm{R} 7)$. Some evaluation methods are proposed for each category in the sequel.

\subsection{Heterogeneous Node Mobility}

Heterogeneity is a key property in PNs [16]. In order to examine the heterogeneous node mobility, we define Mobility Heterogeneity Value (MHV). In PNs, some nodes are static, some nodes move with a person at a nominal walking speed. If the person travels by car, his nodes move much faster than the walking speed. Thus we assume that all the nodes considered have $l$ levels of mobility; in each level, nodes have the same mean speed $v$; the percentage of the number of nodes in each level is $q$. Thus the mean speed of all the nodes, $\bar{V}$, is 


$$
\bar{V}=\sum_{i=1}^{l} v_{i} q_{i}
$$

We define Mobility Heterogeneity Value $(M H V)$ as,

$$
M H V=\sum_{i=1}^{l}\left[\left(\bar{V}-v_{i}\right)^{2} q_{i}\right]
$$

A higher value of $M H V$ means higher heterogeneous node mobility. For all the existing mobility models introduced in Sect. 3, their $M H V$ is zero, because every node has the same minimum and maximum speed. For PNMM, the nodes $(N)$ are separated into two kinds: 'never move' and 'may move' $\left(n_{m}\right)$. Without group merging and splitting, the 'can move' nodes $\left(n_{m}\right)$ have the same mean speed $V_{\max } / 2$, as explained in Sect. 5. Thus $M H V$ of the PNMM is

$$
\mathrm{MHV}_{\mathrm{PNMM}}=\left(\bar{V}_{\mathrm{PNMM}}-\frac{V_{\max }}{2}\right)^{2} \times\left(\frac{n_{m}}{N}\right) .
$$

So $\mathrm{MHV}_{\mathrm{PNMM}}$ is higher, and PNMM represents higher mobility heterogeneity for PNs.

\subsection{Group Mobility and Node Mobility Inside a Group}

To meet R9, nodes inside a group should have relative mobility. We define Relative Mobility in Group $(R M G), \delta$, to describe the relative mobility levels of nodes in a moving group. Firstly, the Average Possible Coverage Area of a Node (APCAN) of node $j, \kappa_{j}^{N}$ is defined as the total area in which a node in a group may possibly reach at any moment during the simulation. We define the Average Possible Coverage Area of a Group (APCAG), $\kappa^{G}$, as the total possible reachable area in which all the nodes in a group move at any moment during the simulation time period. If we assume that $n$ nodes are considered in a group then the Relative Mobility in Group (RMG) is defined as,

$$
\delta=\sum_{j=1}^{n} \kappa_{j}^{N} /\left(n \kappa^{G}\right)
$$

From the above expression we infer that with a bigger $A P C A N, \kappa_{j}^{N}$, of each node in a group w.r.t the $A P C A G, \kappa^{G}$, the relative mobility of nodes in that group $\delta$, is higher.

We calculate $R M G, \delta$, of different group mobility models RPGM, RRGM and PNMM. For RPGM [10], $n$ nodes in a group move randomly in a circular area with $n$ reference points (RP). Thus $\kappa_{j}^{N} \leq \kappa^{G}$. If all the RPs are the same, then $\kappa_{j}^{N}=\kappa^{G}$. So $\delta_{\mathrm{RPGM}} \leq 1$. For RRGM [17] we assume that the destination area of a group is $\kappa^{G d}$ and $\kappa_{j}^{N}=\kappa^{G d}$. However, APCAG of RRGM is bigger than the destination area of a group, $\kappa^{G} \geq \kappa^{G d}$, because nodes with a higher speed arrive at the destination early, while other nodes may still be on the way. Thus $\delta_{\mathrm{RRGM}} \leq 1$. In PNMM, since a node can randomly choose its location in the destination area of the group, $\kappa_{j}^{N}=\kappa^{G}$, and $\delta_{\mathrm{PNMM}}=1$. Thus PNMM has the highest relative node mobility inside a group. 


\subsection{Group Merging and Division}

There are only two group mobility models representing the behavior of group merging and division, which are RRGM and PNMM. RRGM [17] gives two group merging requirements: (a) small groups with a few nodes in each group; (b) groups that pause at the destination for a pre-defined period of time. Since these two requirements are not suitable for PNs as explained in Sect. 2, RRGM cannot be used in PNs.

A method to analyze the dynamics of group merging and division in view of individual node is given here. We define Node Change Rate (NCR) as the number of nodes per second that join or leave a group. If the total simulation time is $T$, the time interval to plan group merging and division is $\alpha$, there are $m$ time intervals, where $m=T / \alpha$, and $n_{m d}^{i}$ represents the number of nodes that join or leave a group in the $i$ th time interval $\alpha$.

$$
\mathrm{NCR}=\frac{1}{m} \sum_{i=1}^{m}\left(n_{m d}^{i} / \alpha\right)
$$

Using NCR as a way of describing the dynamics of group splitting and merging, $N C R$ of PNMM (for Scenario-1 explained in Sect. 5.2) is given by,

$$
\mathrm{NCR}_{\mathrm{PNMM}}=\frac{3 N}{2}\left(\frac{X-Y}{X+Y}\right) .
$$

\subsection{Number of Groups and Number of Nodes in Each Group}

In a PN, a user can organize his nodes into a number of groups for convenience. The number of groups can be decided by the user and these groups can merge and split, which results in the number of groups varying in the range $[Y, X]$. The rules of PNMM ensure that the group number varies in this range. None of the other mobility models consider this. The number of nodes should be pre-specified in each group, for RPGM and RRGM. In PNMM, the number of nodes in each group is randomly decided with different values, which shows the non-uniformity in terms of the size of the groups in PNs.

Some evaluation methods for group mobility models are addressed in this section, which include the heterogeneous node mobility, group mobility and node mobility inside a group, group merging and division and, number of groups and number of nodes in each group. We compare three mobility models in terms of group mobility evaluation. Based on the above evaluation, we get the following results,

- $\quad$ PNMM represents higher mobility heterogeneity for PNs;

- PNMM represents relative node mobility inside a group;

- PNMM represents the dynamics of group merge and division;

- PNMM shows non-uniformity in terms of the size of the groups in PNs.

Thus PNMM proves to be the best fit for satisfying R1-R9. PNMM also has the flexibility to transform it into one of the existing models by carefully selecting values of the defined parameters. Moreover, the group mobility evaluation methods proposed in this section can be used to test any group mobility model proposed in future to address its performance of group movement. 


\section{Applying PNMM in Simulations}

Mobility models are designed for the evaluation of technologies and protocols. Although some protocols proposed for PNs have been evaluated by ad hoc mobility models, little has been done to address their performance in PNs. The proposed PNMM enables protocol evaluation regarding the applicability to PNs. In this section, we apply PNMM in the evaluation of a PN protocol named Personal Network Clustering Protocol (PNCP) [8]. The aim of the evaluation through simulation, in this section, is twofold: for the evaluation of PNMM, the performance difference of PNMM and other mobility models has been tested; for the evaluation of PN protocol, the protocol performance in PN scenarios has been addressed. Through the simulation results, we infer whether a protocol can work well in PNs and consequently, we derive some conclusions to improve the future protocol designs.

Four different mobility models, Random walk mobility model (RWMM) [2], RWP [2], RPGM [10] and PNMM, are compared in the simulation to get their influence on the performance of a protocol in PNs. RWMM and RWP are chosen for the simulation, because they are the most commonly used mobility models for ad hoc networks, and have been used in PN protocol evaluation. Instead of using RRGM, which cannot represent mobility in PNs as explained in Sect. 3 and 6, we use RPMM to represent the group mobility for comparison. In the simulation, PNCP [8] is evaluated, which is particularly designed for PNs. PNCP is used only as an example of a protocol for PNs $[8,13,21,22]$. We note that any other protocol could have been used for comparison. However, considering the relevance of PNCP in the context of PNs, we think it is most appropriate for a detailed simulation study.

Nodes in PNs are geographically separated from each other and organized in different locations, where the nodes in one location can share content, data, applications and resources with each other, and communicate with the outside world. Clustering for personal networks intends to organize these heterogeneous nodes into hierarchical clusters. A clustering scheme, PNCP, has been proposed for PNs [8]. PNCP performs the cluster formation based on heterogeneity of PNs [16] in a distributed way. It forms clusters with a moderate number of nodes, limits the cluster radius to $k$-hops, decreases the change in the cluster composition, extends the average membership time in the clusters providing stability and generates less overhead traffic. PNCP is evaluated in this section using following metrics [8]: 1) Average Master Node Time (AMNT) — defined as the average period of time during which a node plays a central controller role (master); 2) Average Cluster Member Time (ACMT)—defined as the average time a cluster member node stays as a member of a personal cluster; 3) Average Cluster Number (ACN) - defined as the average number of clusters formed in the simulation; 4) Average Cluster Maintenance Load (ACML)—defined as the average number of packets sent or forwarded by each node per second to perform cluster maintenance algorithm. Longer $A M N T$, longer $A C M T$ and lower $A C M L$, yield better performance results for a clustering protocol [8].

PNMM is implemented as a PN mobility generator producing mobility files for the network simulator ns-2 [6]. For all the simulations the ns-2 simulator version 2.31 was used. The parameters used in the simulation are given in Table 3. Other parameters not in Table 3 are the same as those used in the simulation in [8]. For the four mobility models, we set the maximum speed of each mobile node or each group to $3 \mathrm{~m} / \mathrm{s}$ to ensure the similarities of the velocities of nodes in all the models. For RWP, the pause duration of $1 \mathrm{~s}$ is used. For RPMM, we use its implementation in the USC mobility generator tool [23], and there are ten groups in the simulation in which each node has a speed deviation of 10 and an angular deviation of $20^{\circ}$ [23]. For PNMM, the maximum group radius is set to $20 \mathrm{~m}$. The maximum number of 
Table 3 Common set-up for the simulations

\begin{tabular}{llll}
\hline Properties & Values & Properties & Values \\
\hline Terrain dimension & $100 \mathrm{~m} \times 100 \mathrm{~m}$ & Hello interval & $1 \mathrm{~s}$ \\
Simulation time & $100 \mathrm{~s}$ & Cluster size upper limit & 30 nodes/cluster \\
Transmission range & $15 \mathrm{~m}$ & Cluster size lower limit & 5 nodes/cluster \\
Mac layer type & 802.11 & Cluster radius & 2 hops \\
\hline
\end{tabular}
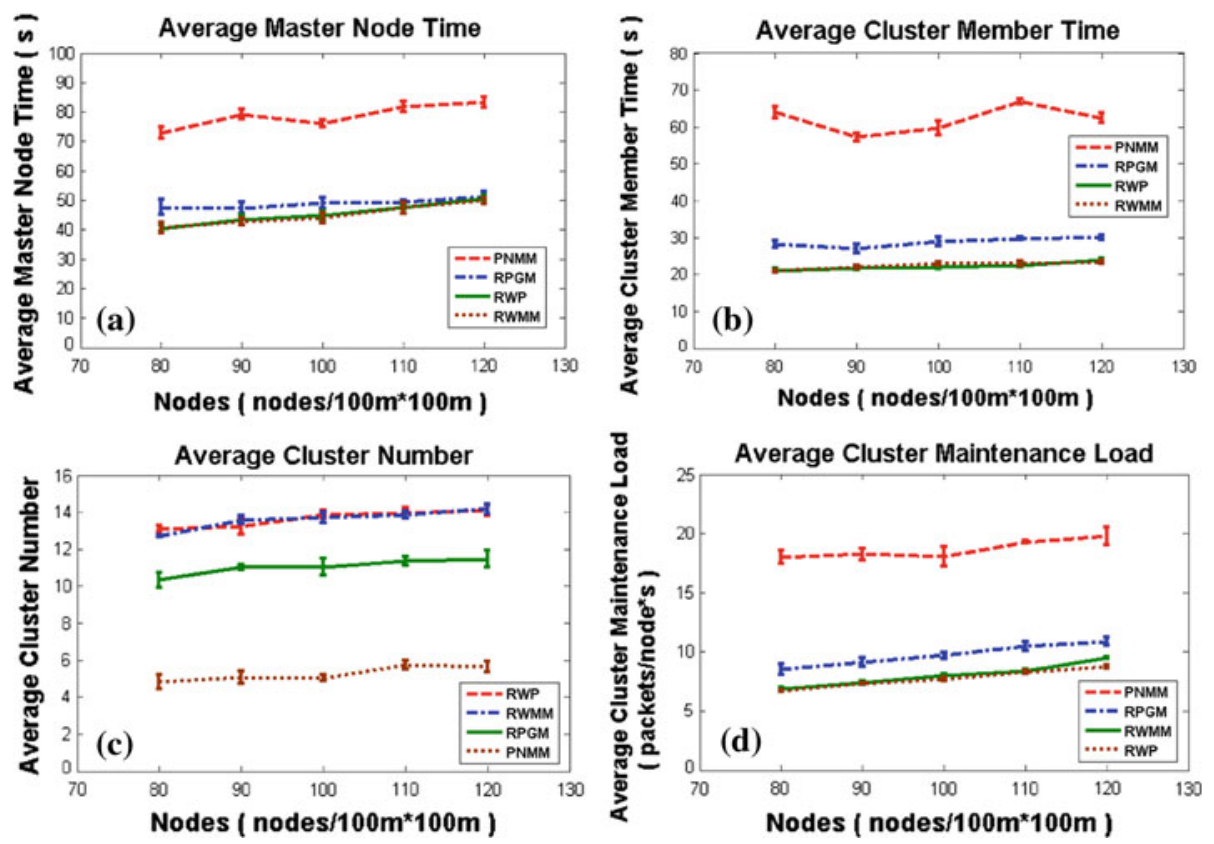

Fig. 8 Performance Comparison of PNMM, RPGM, RWMM and RWP

groups $(X)$ is 10; the minimum number of groups, $(Y)$ is 5 . Ten simulation experiments were performed for each combination to have a mean with a confidence level higher than $95 \%$.

Figure 8a demonstrates the comparison of different mobility models in terms of AMNT. The $A M N T$ in the simulation is almost constant and it increases slowly with the number of nodes. This shows that PNCP can give consistent performance for varying number of nodes. The performance of PNMM is the highest; followed by RPGM; RWP and RWMM perform almost similarly both being the lowest. Thus the performance of PNCP w.r.t. AMNT is better under the PNMM than other mobility models.

Figure $8 \mathrm{~b}$ shows the comparison in terms of $A C M T$, which indicates the stability of the membership in clusters. The ACMT under PNMM case is the highest; RPGM is next; RWP and RWMM yield almost the same performance which is the lowest. Thus, with $A C M T$, PNCP works better under PNMM designed for PNs.

Figure $8 \mathrm{c}$ explains the performance in terms of the $A C N$. The $A C N$ values under PNMM are lower than RWMM and RWP. This significant difference is due to the fact that the nodes move in groups and not in a random manner as assumed in ad hoc networks. PNMM 
significantly influences the performance of PNCP w.r.t. $A C N$, because it represents the PN mobility properties, where nodes move in groups. In addition, the results of RPGM are in the middle, around 10 groups, because the number of groups under RPGM is set as 10 .

The performance in terms of $A C M L$ is demonstrated in Fig. 8d. The $A C M L$ increases with the increase in the number of nodes because there are more neighbors to forward messages in the simulation area. The $A C M L$ under PNMM is higher than the other mobility models. RPGM has lower overhead. RWMM and RWP perform similarly and are the least. Since PNCP under PNMM forms less number of clusters and a higher number of members per cluster, there are more nodes in each cluster forwarding messages [8], which results in higher overhead. Further, the performance of RWMM and RWP are similar because, the only difference between them is being with and without the pause time.

The performance results of PNCP in Fig. 8 show the comparison of the 4 mobility models. PNMM significantly influences the performance and summarized as follows:

- Under PNMM, PNCP performs better in terms of AMNT and ACMT.

- PNMM results in poor performance in terms of ACML, which is expected due to the frequent maintenance.

As shown in Fig. 8, PNCP forms more stable clusters at the cost of higher overhead in PN scenarios than ad hoc networks. PNCP performs differently in PN scenarios compared to its performance in ad hoc networks. Thus the performance of PNCP in PN scenarios needs to be evaluated.

Further examining the performance of the PNCP in different PN scenarios, Fig. 9 demonstrates the performance with three different mobility patterns of PNMM with the maximum group radius of 20,30, and $40 \mathrm{~m}$. Figure $9 \mathrm{a}$ shows the performance w.r.t. AMNT. The performance of PNCP w.r.t. $A M N T$ is better with the group radius of $20 \mathrm{~m}$. As the group radius increases from 20 to $40 \mathrm{~m}$, the $A M N T$ decreases. The larger the value of the maximum group radius, larger is the overlapping area. With larger overlapping area of groups, the $A M N T$ is lower. When the maximum group radius is $40 \mathrm{~m}$, the $A M N T$ is the least. This shows that higher overlapping results in poor performance of PNCP w.r.t. AMNT. Figure $9 \mathrm{~b}$ shows the results of $A C M T$. As maximum group radius increases, the $A C M T$ decreases. Thus the overlapping of groups results in poor performance of clusters vis-à-vis $A C M T$. Figure $9 \mathrm{c}$ explains the performance in terms of $A C N$. With a larger group radius, the number of clusters thus formed is higher because of more overlapping of groups. The performance in terms of $A C M L$ is demonstrated in Fig. 9d. As the number of nodes increase the $A C M L$ increases, because there are more neighbors to forward messages in the simulation area. As the maximum group radius increases from 20 to $40 \mathrm{~m}$, the $A C M L$ slightly decrease. Larger group radius increases the group coverage area and the distance amongst the nodes, which reduces the number of neighbors that can forward the messages [8].

In Fig. 9, PNCP has been tested under different situations described by PNMM, where the group radius is changed from 20 to $40 \mathrm{~m}$. We consolidate results from Fig. 9 here:

- With a larger group radius (more overlapping of groups), AMNT and ACMT are shorter, which means cluster structure is not so stable;

- A larger group radius gives slightly lower overhead produced by PNCP.

Consequently, a larger group radius leads to poor performance of PNCP in terms of cluster stability. Since formation of stable clusters and reduction of re-clustering are the performance design goals for any clustering protocol, a group with larger geographical coverage having more overlap between groups should be considered in the future while designing clustering protocols. When two groups $(A, B)$ are overlapped, a node in Group $A$ if located in the coverage area of Group $B$ may be added to Group $B$. As Group $A$ moves away from Group $B$, 

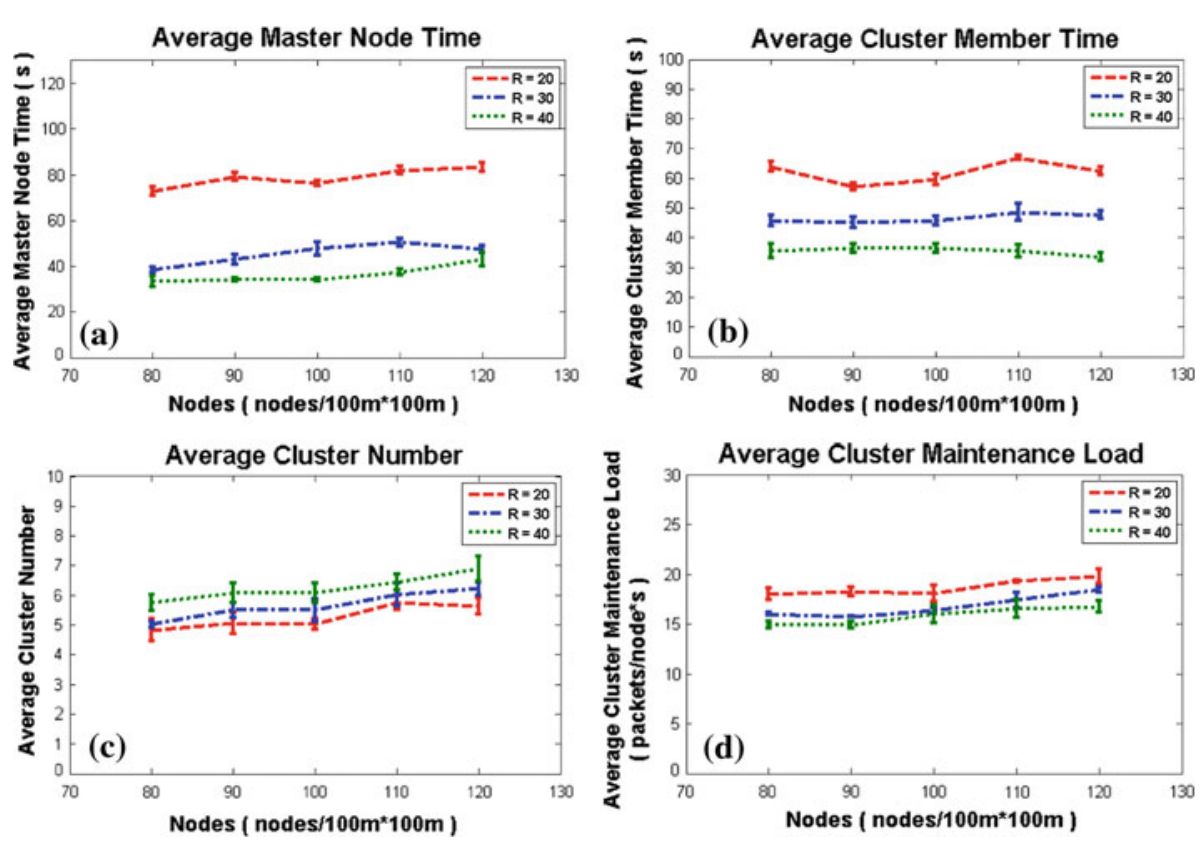

Fig. 9 Performance of PNCP under PNMM

the node will be re-inducted into the Group A. This flip-flop of departing and re-joining of a node into a cluster results in unstable cluster structure. A clustering protocol, which can predict that a particular node will move together with Group A, will reduce the influence of this departing and re-joining. Thus advanced mobility prediction may be a way of solving this problem for a clustering protocol in the future.

In this paper, we generated different mobility patterns by changing the parameters in PNMM. PNCP performs differently in each situation, which shows the design limitation of PNCP. Knowing this limitation, we have to further fine-tune the performance of PNCP. The group radius is one of the parameters that can be changed in PNMM. By changing other parameters of PNMM, such as, simulation area, number of nodes, average speed, etc., various situations in PNs can be generated. To further evaluate the performance of PNCP, more $\mathrm{PN}$ scenarios will be used in the near future.

\section{Concluding Remarks}

It is expected that hundreds of nodes, even thousands of nodes, per person is foreseen in the near future [4,24]. These nodes will work autonomously, individually, and collectively. Personal Network has many possible solutions $[1,8,13,16,18,21,22]$ to interconnect large number of nodes to offer adaptive personal service to the user. The mobility issues of PNs, in such a complex situation have been discussed in this paper. PNMM is proposed and verified. It can produce a steady state, which is verified by our studies. It applies better than other models with respect to the PN mobility properties. It has a significant influence on the performance of protocols designed for PNs. 
From the point of view of various PN services and applications, the behavior of personal nodes is analyzed and the desirable properties of PN node mobility are summarized as Requirements (R1-R9). Based on R1-R9 this paper revisits the prominent mobility models in an ad hoc network and shows that none of them can meet all the requirements. PNMM proposed here has a stable long-run behavior and provides a steady state, which is proved by the stability analysis and simulation of PNMM. From stability study we get in-depth details of the performance of PNMM such as, groups merging and splitting, change in the average speed of groups and the average speed of nodes. A larger simulation area increases the average number of groups. For mobility model evaluation, the performance differences of mobility models were studied by Musolesi et al. [14]. However, it is insufficient to verify whether a mobility model can represent a certain scenario. We proposed some mobility modeling evaluation methods to find the extent a mobility model can represent the properties of the target scenario. Some examples are node mobility heterogeneity-Mobility Heterogeneity Value (HMV); Relative Mobility in Group (RMG); and dynamics of group merging and splitting-Node Change Rate (NCR). These evaluation methods show the way to test how realistic they are in describing the mobility properties in real-life situations. The comparison of the four mobility models-RWMM [2], RWP [2], RPGM [10] and PNMM-shows that PNMM significantly influences the performance of the protocols designed for PNs. Furthermore, we applied PNMM in the evaluation of a PN protocol, named Personal Network Clustering Protocol (PNCP) [8]. PNCP was evaluated using RWP presenting general ad hoc network scenarios and PNMM describing PN scenarios. The simulation results show that PNMM perform differently in ad hoc networks and PNs. Thus techniques and protocols proposed for PNs should be evaluated under PNMM to test whether they can perform in PNs presented by PNMM similar to the ad hoc environments.

The performance of PNCP is further evaluated by various scenarios presented by PNMM. These performance results provide insights and design guidelines for future improvement of clustering protocols for PNs. Skeptics may question the validity of applying PNMM for evaluation of PNCP. PNMM is, after all, expected to be better since it is designed for PNs. However, we stress here the fact that PNCP is a generalized clustering protocol and it can be applied to any heterogeneous group of nodes. PNCP, in a homogeneous environment would be similar to many other clustering protocols found in the literature [8].

PNMM in general proposes some guidelines in modeling mobility in PNs. The future design of mobility modeling should be close to reality. PNs involve personal nodes in heterogeneous networks. Considering the heterogeneity of wireless access technologies proposed in [26] is a way of improving the design of mobility models for heterogeneous networks such as PNs. The impact of environment is another direction of future mobility modeling. The fixed paths to destinations, obstacles and other nodes, and hotspots (high density of nodes in certain places) are examples of environmental factors influencing the movement of nodes, which will be further studied for PN mobility modeling. Moreover, using the real mobility traces of personal nodes is of course an efficient way for the design and evaluation of PN mobility models. Although the real data is not available at this juncture, it would further improve the PN mobility model.

The proposed PNMM can be used to evaluate the protocols and techniques for PNs, such as self-organization [13], context awareness [21], service discovery and management [22], etc. Although most of these protocols and techniques have been tested under ad hoc mobility models, there is a difference in the performance between PNMM and other mobility models as shown in Sect. 7. Whether these protocols and techniques can work well under PNMM is still unknown. With more tests, the performance thus found would be useful in the further improvement of protocols and techniques for PNs. Moreover, by considering the 
unique properties of mobility in PNs analyzed in this paper, the protocols and techniques designed for PNs should be equipped with some special mechanisms to fit the PN scenarios. A major contribution of this work is that we can use these results in planning/studying PANs and Body Area Networks (BANs) too. Since the PNs are an extension of PANs and BANs. The immediate task in hand for us is to validate PNMM through implementation on our pilot test bed.

Acknowledgments This work is partially funded by the EU IST My personal Adaptive global NET (MAGNET) Beyond Project and the Dutch Freeband Personal Network Pilot 2008 (PNP2008) Project and STW Future Home Networks.

Open Access This article is distributed under the terms of the Creative Commons Attribution Noncommercial License which permits any noncommercial use, distribution, and reproduction in any medium, provided the original author(s) and source are credited.

\section{References}

1. Calin, D., McGee, A. R., Chandrashekhar, U., \& Prasad, R. (2006). MAGNET: An approach for secure personal networking in beyond 3G wireless networks. Bell Labs Technical Journal, 11(1), 79-98.

2. Camp, T., Boleng, J., \& Davies, V. (2002). A survey of mobility models for ad hoc network research. In Proceedings of the wireless communication \& mobile computing (WCMC), special issue on mobile ad hoc networking: research, trends and applicatons, 2(5), pp. 483-502.

3. Charas, P. et al. (2000). The book of visions: Visions of the wireless world. Wireless strategic initiative.

4. Colin, J. R. (2008). The future according to freescale: 1000 embedded devices per person, EE Times, http://www.eetimes.com/showArticle.jhtml?articleID:208700500. Accessed 25 Nov 2008.

5. Ducatel, K. et al. (2001). Scenarios for ambient intelligence in 2010. IST advisory group (ISTAG), European Commission.

6. Fall, K., \& Varadhan, K. (2005). The ns Manual, http://www.isi.edu/nsnam/ns/doc.pdf.

7. Flament, M. et al. (1998). Telecom scenarios in 2010-A wireless infrastructure perspective. http:// citeseerx.ist.psu.edu/viewdoc/summary? doi:10.1.1.28.7750. Accessed 25 Nov 2008.

8. Gu, Y., Lu, W., Prasad, R. V., \& Niemegeers, I. G. M. M. (2007). Clustering for ad hoc personal network formation. In Proceedings of the international conference on computational science (ICCS), Beijing.

9. Gu, Y., Prasad, R. V., \& Niemegeers, I. G. M. M. (2008). A mobility model for personal networks. In Proceedings of the 7th annual mediterranean ad hoc networking workshop (Med Hoc Net 2008).

10. Hong, X., Gerla, M., \& Chiang, C. (1999). A group mobility model for ad hoc wireless networks. In Proceeding of the ACM international workshop on modelling and simulation of wireless and mobile systems (MSWiM).

11. Hui, P., Chaintreau, A., Scott, J., Gass, R., Crowcroft, J., \& Diot, C. (2005). Pockets switched networks and human mobility in conference environments. In Proceedings of the ACM SIGCOMM'05 (pp. 244-251).

12. Johnson, D., \& Maltz, D. (1996). Dynamic source routing in ad hoc networks. In Mobile computing (pp. 153-181).

13. Lu, W., Gu, Y., Prasad, R. V., Lo, A., \& Niemegeers, I. G. M. M. (2007). A self-organized personal network architecture. In Proceedings of the 3rd international conference on networking and services (ICNS'07).

14. Musolesi, M., \& Mascolo, C. (2006). A community based mobility model for ad hoc network research. In Proceedings of the REALMAN'06, Florence, Italy.

15. Navidi, W., \& Camp, T. (2004). Stationary distribution for the random waypoint mobility model. IEEE Transactions on Mobile Computing, 3(1).

16. Niemegeers, I. G. M. M., \& de Groot, S. M. H. (2003). Research issues in ad-hoc distributed personal networking. Journal of Wireless Personal Communications, 26(2-3), pp. 149-167, Kluwer Academic Publishers.

17. Ng, J. M., \& Zhang, Y. (2005). Reference region group mobility model for ad hoc networks. In Proceeding of wireless and optical communications networks (WOCN).

18. Prasad, R., \& Skouby, K. (2005). Personal Network (PN) applications. Journal of Wireless Personal Communications, 33, pp. 227-242, Springer. 
19. Royer, E., Melliar-Smith, P. M., \& Moser, L. (2001). An analysis of the optimum node density for ad hoc mobile networks. In Proceedings of the IEEE international conference on communications (ICC).

20. Sanchez, M., \& Manzoni, P. (2001). A java based simulator for ad-hoc network. Future generation computer systems, pp. 573-583.

21. Sanchez, L., Lanza, J., Olsen, R., Bauer, M., \& Girod-Genet, M. (2006). A generic context management framework for personal networking environments. In Proceedings of the 3rd annual international conference on mobile and ubiquitous systems: networking \& services.

22. Stephen, H., \& Aruna, S. (2006). Service composition for mobile personal networks. In Proceedings of the 3rd annual international conference on mobile and ubiquitous systems: networking \& services.

23. USC mobility generator tools, http://nile.usc.edu/important/software.htm.

24. Weiser, M. (1996). Ubiquitous computing. http://www.ubiq.com/hypertext/weiser/UbiHome.html. Accessed 25 Nov 2008.

25. Yoon, J., Liu, M., \& Noble, B. (2003). Random waypoint considered harmful. In Proceeding of 21st Ann. Joint Conf. IEEE Computer and Comm. Soc. (INFOCOM 2003) (pp. 1312-1321).

26. Zahran, H., Liang, B., \& Saleh, A. (2008). Mobility modeling and performance evaluation of heterogeneous wireless networks. IEEE Transactions on Mobile Computing, 7(8), 1041-1056.

\section{Author Biographies}

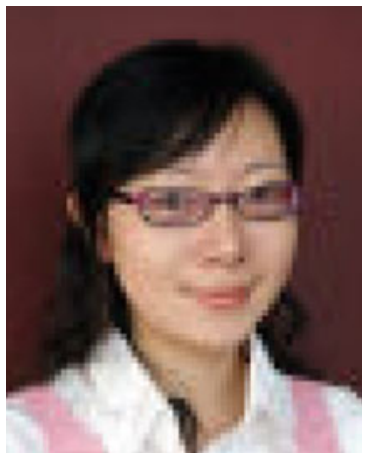

Yanying Gu was born in Shenyang, China in 1981. She received her Bachelors degree in the Department of Electronic Engineering in Dalian University of Technology, Dalian, China in 2004. She received her Masters degree in the Department of Telecommunications in Delft University of Technology, Delft, The Netherlands in 2006. She is a Ph.D. student at Delft University of Technology, The Netherlands. She is interested in working in research groups, contributing her fresh ideas, creativities and passion. Currently her research interests include personal networks, clustering protocols in wireless ad hoc networks, routing protocols in wireless ad hoc networks, context-aware intelligence, and indoor position sensing techniques.

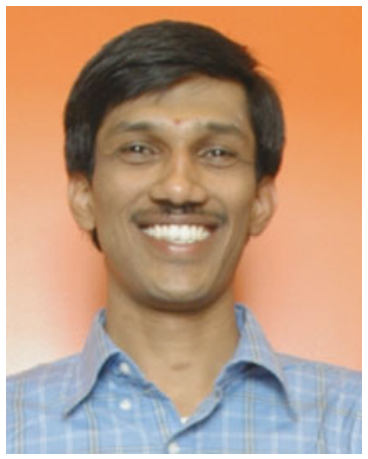

R. Venkatesha Prasad received bachelors degree in Electronics and Communication Engineering from the University of Mysore, India, in 1991. In 1994 he received a M.Tech. degree in Industrial Electronics and a Ph.D. degree in 2003 from University of Mysore, India and Indian Institute of Science, Bangalore, India. During 1994 and 1996 he was working as a consultant and project associate for a private firms and ERNET lab of ECE Department in Indian Institute of Science. While pursuing Ph.D., from 1999 to 2003, he was working as a consultant for CEDT, Indian Institute of Science, for VoIP application development as part of Nortel Networks sponsored project. From 2003 to 2005 he was heading a team of engineers at the Esqube Communication Solutions Pvt. Ltd., Bangalore, India, for the development of various networking solutions. From 2005 until date he is with the Wireless and Mobile Communications Group, Delft University of Technology, the Netherlands, working on the EU funded projects MAGNET, MAG-

NET Beyond and PNP 2008. He is part of TPC of many IEEE conferences and a regular reviewer for many journals. He has three patents and five under review, and published many articles and also is part of two IEEE standards. He is also IEEE 1900 Standardization committee member and member of IEEE TCCN and AHSNTC. Currently he is a consultant for Esqube communications, Bangalore, India, working on innovative voice enabling applications. 


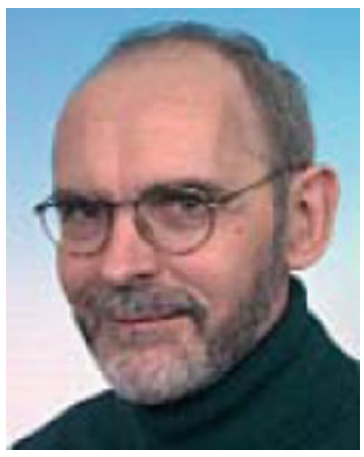

Ignas Niemegeers received a degree in Electrical Engineering from the University of Gent, Belgium, in 1970. In 1972, he received an MSEE degree in Computer Engineering and in 1978 a Ph.D. degree from Purdue University in West Lafayette, Indiana, USA. From 1978 to 1981 he was a designer of packet switching networks at Bell Telephone Mfg. Cy, Antwerp, Belgium. From 1981 to 2002 he was a professor in the Faculty of Computer Science and Electrical Engineering at the University of Twente, Enschede, The Netherlands. From 1995 to 2001, he was Scientific Director of the Centre for Telematics and Information Technology (CTIT) of the University of Twente, a multi-disciplinary research institute in ICT and applications. Since May 2002, he holds the chair in Wireless and Mobile Communications at Delft University of Technology in The Netherlands, where he is heading the Centre for Wireless and Personal Communications (CWPC) and the Department of Telecommunications. He is an active member of the Wireless World Research Forum (WWRF) and IFIP TC-6 Working Group on Personal Wireless Communications. He has been involved in many European research projects, in particular ACTS TOBASCO, ACTS PRISMA, ACTS HARMONICS, RACE MONET, RACE INSIGNIA and RACE MAGIC. Presently, he is participating in the IST projects MAGNET on personal networks, and EUROPCOM on emergency ad hoc networks. 